CLINICAL STUDY

\title{
The CC genotype of the GNAS T393C polymorphism is associated with obesity and insulin resistance in women with polycystic ovary syndrome
}

\author{
Susanne Hahn, Ulrich H Frey ${ }^{1}$, Winfried Siffert ${ }^{1}$, Susanne Tan ${ }^{2}$, Klaus Mann ${ }^{2}$ and Onno E Janssen ${ }^{2}$ \\ Endokrinologikum Ruhr, Center for endocrine and metabolic diseases, Alter Markt 4, 44866 Bochum, Germany, ${ }^{1}$ Institute of Pharmacogenetics, and \\ ${ }^{2}$ Division of Endocrinology, Department of Medicine, University Hospital of Essen Medical School, Essen, Germany \\ (Correspondence should be addressed to S Hahn; Email: susanne.hahn@endokrinologikum.com)
}

\begin{abstract}
Objective: Variants in the Gs protein $\alpha$ subunit gene (GNAS1) are known to be involved in the pathogenesis of several endocrine and metabolic disorders. To understand genetic determinants of androgen excess, insulin resistance, and obesity in polycystic ovary syndrome (PCOS), we investigated the effect of the common GNAS1 T393C polymorphism on the phenotype of German PCOS women.

Design: Two hundred and seventy-eight PCOS women and 820 Caucasian controls were genotyped for the common T393C polymorphism in GNAS1. To this end, genomic DNA was amplified by PCR with specific oligonucleotides and genotypes were determined using the restriction enzyme FokI. In addition, we evaluated whether the T393C polymorphism had an influence on the response to 6 months metformin treatment in a subgroup of 105 PCOS women. Methods: Anthropometric variables, metabolic parameters including indices of insulin resistance measured by oral glucose tolerance testing, and endocrine biochemical as well as clinical parameters were measured in all PCOS subjects.

Results: GNAS1 genotype distributions were not significantly different between PCOS women and controls. In PCOS women, no significant differences in endocrine clinical and biochemical variables were found between the genotypes. However, the C-allele carrier group had significantly higher mean body weight, body mass index, leptin levels, and higher indices of insulin resistance compared with women with GNAS1 TT-genotype. Metformin treatment significantly improved hyperandrogenism, menstrual cyclicity, body weight, and insulin resistance independent of GNAS1 genotype. The major determinant of weight loss was body weight before treatment. Conclusion: The T393C polymorphism is not associated with PCOS in Caucasian women, but may represent a genetic marker for increased susceptibility for obesity in this cohort.
\end{abstract}

European Journal of Endocrinology 155 763-770

\section{Introduction}

Polycystic ovary syndrome (PCOS) is among the most common endocrine disorders, affecting more than $5 \%$ of reproductive aged women. Family studies support a familial aggregation of PCOS (1), consistent with a genetic basis for this disorder. Associations of PCOS with mutations in a variety of candidate genes involved in pathways regulating steroid hormone synthesis $(2,3)$ or insulin metabolism have been analyzed, without evidence of a single common defect. In women with PCOS, the impairment of insulin metabolism is thought to be influenced by both environmental (4) and genetic factors $(5,6)$. Recently published studies from the Czech Republic (7) and Finland (8) have shown that insulin resistance and metabolic abnormalities are related to obesity rather than to PCOS per se. Obesity is a common feature of women with PCOS in different ethnic cohorts affecting about half of the entire PCOS population $(9,10)$.

Genetic polymorphisms predisposing to obesity are of major interest. It is well known that obesity is due to environmental factors and lifestyle, but a great amount of body mass variation is likely to be inherited (11). Defects in the melanocortin pathway are related to severe obesity (12). Similar results were obtained for leptin receptor mutations (13), variations of the resistin gene (14), and the adiponectin locus (15). In Germans, Chinese, and South Africans, the $825 \mathrm{~T}$ allele of the gene GNB3 encoding the G-protein $\beta 3$ subunit is significantly associated with obesity with odds ratios (ORs) between 2 and 3 (16). In PCOS women, the microsatellite CA-repeat polymorphism in the interleukin $6 R-\alpha$ gene was shown to influence obesity (17). 
A possible approach to identify susceptibility genes for obesity or PCOS per se is to test the association between clinical characteristics and a specific allele of a gene that appears to be a good candidate gene, for example, a gene involved in hormone production and insulin metabolism, like the gene GNAS1 encoding for the Gs protein $\alpha$ subunit (Gas), which is located at 20q13.2-13.3, a locus that has previously been linked to obesity (18). Gas, which couples receptor binding by several hormones to activation of adenylate cyclase, is one member of a large family of G-proteins that are integral components of diverse signaling pathways (19). In mice, the arrest of meiotic prophase oocytes within antral follicles requires the G-protein $\mathrm{G}(\mathrm{s})$ and the orphan Gs-linked receptor GPR3 (20-22). In humans, several endocrine disorders are associated with somatic GNAS1 mutations $(23,24)$. In young girls with premature thelarche, the R201H-activating mutation in the GNAS1 was suggested to cause ovarian hyperfunction (25). In addition, the Gas protein system has been shown to play an important role in the metabolic and the cardiovascular system. In animal models, mice with heterozygous disruption of the GNAS paternal allele are lean, hypermetabolic, and present with an increased whole body insulin sensitivity (26). In humans, heterozygous Gas null mutations lead to Albright hereditary osteodystrophy, a syndrome, among other symptoms, characterized by obesity (27). Genetic variation of G-proteins may play an important role not only in complex diseases, but also in drug responses. Jia et al. hypothesized that the GNAS1 locus carries a functional variant influencing the response to $\beta$-blockade in hypertensive patients (28). Interactions between genes and therapeutic interventions were also found for several other genes. In PCOS women, Ertunc et al. found a differential effect of metformin therapy on the basis of IRS genotypes (29).

To understand genetic determinants of androgen excess, insulin resistance, and obesity in PCOS women, we investigated the effect of the common GNAS1 T393C polymorphism on the phenotype of German PCOS women. In addition, we evaluated whether genotypes of the T393C polymorphism had an influence on the response to metformin treatment.

\section{Subjects and methods}

\section{Subject recruitment}

PCOS patients From the outpatient clinics of the Division of Endocrinology, Department of Medicine and the Department of Obstetrics and Gynecology at the University Hospital of Essen Medical School, 278 PCOS patients seeking medical advice for cycle abnormalities, hirsutism, obesity, or infertility were prospectively and consecutively recruited. Based on the criteria derived from the $1990 \mathrm{NIH}$ conference, diagnosis of PCOS was established when either oligomenorrhea (cycles lasting longer than 35 days) or amenorrhea (less than two menstrual cycles in the past 6 months) and either clinical signs of hyperandrogenism (hirsutism or obvious acne or alopecia and/or an elevated total testosterone in combination with an elevated free androgen index (FAI) (normal range: testosterone $<2.0 \mathrm{nmol} / \mathrm{l}, \mathrm{FAI}<3.8$ ) were found, and other pituitary, adrenal, or ovarian diseases could be excluded. Therefore, laboratory analysis of luteinizing hormone (LH), follicle-stimulating hormone (FSH), estradiol, prolactin, cortisol, adrenocorticotropic hormone (ACTH), thyroid-stimulating hormone (TSH), insulin-like growth factor (IGF)-1, androstendione, and DHEA sulfate was performed in each woman. In addition, in all women an ACTH test with measurement of 17-hydroxyprogesterone was performed. When the stimulated value was > $10 \mathrm{ng} / \mathrm{ml}$, a genetic analysis (21 hydroxylase deficiency) was added. Hirsutism was routinely graded by two physicians independently using the common modified Ferriman-Gallwey score (FG). FG scores never differed by more than 2 and when not identical were re-evaluated by a third physician and the median value used. Hirsutism was diagnosed when a score above 5 was evaluated. All PCOS women also fulfilled the 2003 Rotterdam criteria (30). PCOS women were not taking any medication known to affect carbohydrate and lipid metabolism or endocrine parameters for at least 3 months before entering the study. All PCOS women were of Caucasian origin. The study protocol was approved by the Ethics Committee of the University of Essen. Written informed consent was obtained from all participants.

Control population The Caucasian control sample consisted of 820 healthy white blood donors, who were recruited at the local Department for Transfusion Medicine, University Hospital of Essen.

These individuals attend the blood bank usually every 3 months for blood donation and are under close health surveillance to guarantee high-quality blood products. According to German law, blood donors must be free of any medication and acute or chronic infectious diseases, to mention only few requirements. Thus, these individuals represent a cross-sectional sample of a young healthy German population. Among these 820 controls, healthy white women were matched for race and age with PCOS women. The 209 female age-matched blood donors had a mean age of $29.4 \pm 6.2$ years.

\section{Study protocol}

A subgroup of 124 PCOS women not wishing to take oral contraceptives received monotherapy with metformin. This sample was comparable with regard to the 
clinical, endocrine, and metabolic characteristics to a larger sample of German PCOS patients, which we have recently described in detail (10). Women with body mass index $(\mathrm{BMI})<30 \mathrm{~kg} / \mathrm{m}^{2}$ were treated with $850 \mathrm{mg}$ bid and those with BMI $\geq 30 \mathrm{~kg} / \mathrm{m}^{2}$, with $1000 \mathrm{mg}$ bid. The study protocol was approved by the Ethics Committee of the University of Duisburg-Essen. All participants gave informed written consent before entering the study. In addition, a patient's insurance was contracted for all study participants treated with metformin.

PCOS women were told not to change their lifestyle behavior (nutrition, sport) during the study period in order to make sure that a possible weight loss is only induced by metformin therapy. Patients and physicians were unaware of patients' genotypes at any time of the study period. Out of the 124 patients, 15 PCOS women conceived during the study and were excluded from further analysis. Three women discontinued treatment because of side effects (diarrhea) and one was excluded from the analysis because she wished to take an oral contraceptive pill. Therefore, data of 105 women were available for statistical analysis.

\section{Clinical and laboratory parameters}

In PCOS subjects, physical examination was performed, including evaluation of hirsutism by the FG-score, the presence of acne or alopecia, and anthropometric measurements including body weight in kilograms and waist circumference in centimeters before entering the study $(n=278)$ and in those participating in the treatment subgroup $(n=105)$ again after 1 and 6 months on metformin. Body mass index (BMI) was calculated as weight $/(\text { height })^{2}\left(\mathrm{~kg} / \mathrm{m}^{2}\right)$. Sitting blood pressure was measured twice after a 15 -min rest from the right arm using a standard sphygmomanometer, while the appearance of the first sound (Korotkoff sound, phase I) was used to define systolic blood pressure and the disappearance of sound (phase V) defined diastolic blood pressure. We used the average of the two measurements for statistical analysis. In addition, medical history was obtained by personal interview. PCOS women were instructed to document the frequency and length of menstrual bleedings. After an overnight fast of $12 \mathrm{~h}$, a 75 g-oral glucose tolerance test (OGTT) with determination of glucose and insulin levels at baseline and at 30, $60,90,120$, and $180 \mathrm{~min}$ was performed. Insulin resistance was defined by the HOMA-model (31) and insulin sensitivity by evaluating the quantitative insulinsensitivity check index (QUICKI) (32) and the Bennett index (33). In addition, whole-body insulin sensitivity (ISI ${ }_{\text {Matsuda }}$ (34), which combines hepatic and peripheral insulin sensitivity, was calculated by the formula: 10 000/square root of [(fasting glucose $\times$ fasting insulin $) \times($ mean glucose $\times$ mean insulin during OGTT (times 0, 30, 60, 90, and $120 \mathrm{~min})]$. Hyperinsulinemia was determined by calculating the area under the insulin response curve (AUC-I) and $\beta$-cell function by HOMA- $\beta$ (31). Prior to the OGTT (between 0800 and $0900 \mathrm{~h}$ ), blood samples were drawn for the measurement of all other metabolic and endocrine parameters. Except for amenorrhoic women, all laboratory determinations were performed in the early follicular phase of the cycle. The presence of polycystic ovaries (PCO) was defined by the ESHRE/ASRM-criteria when at least one ovary $>10 \mathrm{ml}$ or with at least 12 follicles of 2-9 mm diameter was found on transvaginal ultrasound. FAI was estimated as testosterone $(\mathrm{nmol} / \mathrm{l}) / \mathrm{sex}$ hormone-binding globulin $(\mathrm{SHBG} ; \mathrm{nmol} / \mathrm{l}) \times 100$.

Automated chemiluminescence immunoassay systems were used for the determination of testosterone, LH, FSH, estradiol, prolactin, cortisol, TSH, total cholesterol, HDL cholesterol (HDL), LDL cholesterol (LDL), triglycerides, and blood glucose (ADVIA Centaur, Bayer Vital, Fernwald, Germany), androstendione, DHEA sulfate (DHEAS), SHBG, and insulin (IMMULITE 2000, DPC Biermann, Bad Nauheim, Germany). Leptin was measured using the enzymatically amplified 'twostep' sandwich-type ACTIVE Human Leptin ELISA kit (Diagnostic Systems Laboratories, Webster, TX, USA). Biologically active ghrelin was determined by RIA kits (LINCO Research, Inc., St. Charles, MO, USA). Intraand interassay variations were $<5$ and $<8 \%$ respectively for all measured parameters.

\section{DNA genotyping}

For the molecular analysis of the GNAS1 alleles, genomic DNA was isolated from whole blood with the QIAmp DNA blood kit (Qiagen, Hilden, Germany). Genotypes for the GNAS1 T393C polymorphism were determined by PCR with the forward primer $5^{\prime}$-CTCCTAACTGACATGGTGCAA- $3^{\prime}$ and the reverse primer 5'-TAAGGCCACACAAGTCGGGGT-3'. After denaturation at $94^{\circ} \mathrm{C}, 35$ cycles of DNA amplification were performed using Eppendorf Taq PCR Mastermix at $94{ }^{\circ} \mathrm{C}$ for $45 \mathrm{~s}, 58^{\circ} \mathrm{C}$ for $40 \mathrm{~s}$, and $72{ }^{\circ} \mathrm{C}$ for $45 \mathrm{~s}$. The $345 \mathrm{bp}$ PCR products were digested using the restriction enzyme FokI and analyzed on a $2 \%$ agarose gel. The unrestricted products (345 bp) represent the TT genotype; the completely restricted products (259 and $86 \mathrm{bp}$ ) represent the CC genotype.

\section{Statistical analysis}

Data are presented as mean \pm s.D. or as number and percent affected. Wherever continuous variables were compared at baseline, linear ANOVA was applied. For not normally distributed variables, Wilcoxon rank sum test (in case of two groups) or Kruskal-Wallis test (in case of more than two groups) was performed.

The Tukey honestly significant difference (HSD) post hoc test was used to determine the significant differences between group means in an ANOVA setting. Tukey's HSD test is one of the several methods of ensuring that 
the chance of finding a significant difference in any comparison (under a null model) is maintained at the $\alpha$ level of the test and therefore preserves 'family-wise type I error'. Since the T393C polymorphism shows a genedose effect (35), ANOVA was used for comparison of continuous variables where appropriate. For categorical variables at baseline, the $\chi^{2}$ test was used; for dichotomous clinical parameters before and after metformin treatment, McNemar test for frequency distributions was calculated. To address treatment response with respect to baseline, comparisons were carried out using Wilcoxon sign rank tests or the mixed model ANOVA for repeated measures where appropriate. Differences were regarded significant at a $P$ value $<0.05$. All statistical analyses were done using SPSS version 11.0 (SPSS, Chicago, IL, USA). Box-plot graphs were computed using GraphPad Prism 4 (GraphPad Software, Inc., San Diego, CA, USA). All data are presented in SI units. Our study had more than $80 \%$ (0.82 802) statistical power to detect an OR of 1.5 with the type 1 error rate $\alpha=0.05$.

\section{Results PCOS characteristics}

Complete baseline data sets were available for a total of 278 PCOS women. The majority of these women were oligomenorrhoic (196 out of $278,71 \%$ ), the rest was amenorrhoic. Regarding clinical signs of hyperandrogenism, 36\% of PCOS subjects presented with acne, $29 \%$ with mild alopecia (Ludwig score $<2$ ) and $67 \%$ with hirsutism. Mean FG hirsutism score was $9.7 \pm 6.7$. Elevated total testosterone levels and FAI were found in $83.1 \%$ (mean testosterone, $2.6 \pm 0.9 \mathrm{nmol} / \mathrm{l}$; mean FAI, $8.8 \pm 6.9$ ) of PCOS patients. PCO, defined by the ESHRE/ASRM-criteria, were diagnosed in 208 women $(75 \%)$. Insulin resistance (HOMA-IR $>2.5$ ) was diagnosed in 187 out of 278 women (67.3\%) (mean, $3.9 \pm$ 3.6). Of the entire PCOS cohort, 87 (31.3\%) women had normal weight $\left(\mathrm{BMI}<25 \mathrm{~kg} / \mathrm{m}^{2}\right), 55(19.8 \%)$ overweight $\left(\mathrm{BMI}=25.0-29.9 \mathrm{~kg} / \mathrm{m}^{2}\right)$, and $136(48.9 \%)$ obese (BMI $\left.\geq 30.0 \mathrm{~kg} / \mathrm{m}^{2}\right)$.

\section{Genotype distribution}

In PCOS women, GNAS1 genotypes did not differ from those evaluated in a control population of 820 randomly selected blood donors (women and men) or from an agematched group of healthy Caucasian women (Table 1). Genotype distributions of both PCOS patients and controls were in Hardy-Weinberg equilibrium.

\section{Patient characteristics by GNAS1 genotype}

In PCOS women, no significant differences in endocrine variables were found between the T393C GNAS1 genotypes (Table 2). However, statistical analysis by linear ANOVA revealed that PCOS CC genotypes had
Table 1 GNAS1 genotype distribution in polycystic ovary syndrome (PCOS) women and controls. Data are presented as number and (percentage).

\begin{tabular}{|c|c|c|c|c|}
\hline & $\mathrm{CC}$ & TC & TT & $n$ \\
\hline PCOS women & $73(26.3 \%)$ & $136(48.9 \%)$ & $69(24.8 \%)$ & 278 \\
\hline $\begin{array}{l}\text { Controls (women } \\
\text { and men) }\end{array}$ & $235(28.7 \%)$ & $403(49.1 \%)$ & $182(22.2 \%)$ & 820 \\
\hline $\begin{array}{l}\text { Age matched } \\
\text { control women }\end{array}$ & 57 (27.3\%) & 95 (45.4\%) & $57(27.3 \%)$ & 209 \\
\hline
\end{tabular}

significantly higher mean body weight, BMI, leptin levels, and higher indices of insulin resistance compared with women possessing GNAS1 TC or TT genotype (Table 3). Differences in body weight, BMI, and leptin levels between the CC and TT genotypes remained significant even after correcting by Tukey's testing. Comparison of the homozygous CC and TT groups of PCOS women also found significant differences in waist circumference, $\beta$-cell function measured by HOMA- $\beta$ and for the Matsuda index as a parameter of combined hepatic and peripheral insulin sensitivity. Significant differences in the genotype distribution according to BMI values were found $(P=0.01)$. The $393 \mathrm{C}$ allele frequency was found to be $73.6 \%$ in normal weight women, $69.1 \%$ in overweight women, $60.9 \%$ in women with a BMI $=30.0-34.9 \mathrm{~kg} / \mathrm{m}^{2}, 85.7 \%$ in women with a BMI $=35.0-39.9 \mathrm{~kg} / \mathrm{m}^{2}$, and $89.6 \%$ in women with a BMI $\geq 40 \mathrm{~kg} / \mathrm{m}^{2}$. Lipid and glucose metabolism were not influenced by the T393C GNAS1

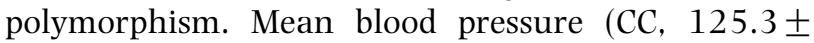
$16.2 \mathrm{mmHg}$; TC, $125.3 \pm 14.4 \mathrm{mmHg}$; TT, $124.6 \pm$ $14.9 \mathrm{mmHg}$ ) and smoking status (CC, 33\% smokers; TC, $30 \%$ smokers; and TT, $44 \%$ smokers) did not differ significantly between the groups. Interestingly, the percentage of PCOS women, who were physically active (sport activities at least once in a week for more than $30 \mathrm{~min}$ ) did not differ significantly between the subgroups (CC, 54.8\%; TC, 52.9\%; TT, 59.4\%).

Control characteristics by GNAS1 genotype. In the age-matched control group of Caucasian women $(n=209)$, no significant differences in the genotype distribution according to body weight (CC, $73.3 \pm 13.3$; TC, $73.6 \pm 11.1$; TT, $75.7 \pm 11.5 \mathrm{~kg}$ ), BMI (CC, $26.2 \pm$ 4.5; TC, $25.3 \pm 3.4$; TT, $26.4 \pm 3.7 \mathrm{~kg} / \mathrm{m}^{2}$ ), or waist circumference (CC, 81.1 \pm 12.5 ; TC, $78.6 \pm 12.8$; TT, $80.4 \pm 12.2 \mathrm{~cm}$ ) were found.

\section{Metformin treatment}

Patients and physicians were unaware of patients' genotypes at any time of the study period. The genotype distribution of the 105 women treated with metformin was not statistically different from that of the entire PCOS cohort (CC, $n=28$ (26.7\%); TC, $n=51$ (48.6\%), TT, $n=26(24.8 \%))$. Metformin therapy significantly improved FAI (Fig. 1) as a parameter of biologically active testosterone as well as total testosterone levels (CC, $2.8 \pm$ $1.0-1.9 \pm 0.8 \mathrm{nmol} / \mathrm{l} ; \quad \mathrm{TC}, 2.7 \pm 1.0-1.9 \pm 0.7 \mathrm{nmol} / \mathrm{l}$; 
Table 2 Endocrine characteristics of PCOS women by GNAS1 genotype. Values are presented as mean and S.D. or number and percentage of affected.

\begin{tabular}{|c|c|c|c|c|}
\hline & $\operatorname{CC}(n=73)$ & TC $(n=136)$ & TT $(n=69)$ & $\boldsymbol{P}$ \\
\hline Testosterone (nmol/l) & $2.6 \pm 0.9$ & $2.6 \pm 0.9$ & $2.6 \pm 0.8$ & NS \\
\hline FAI & $8.4 \pm 5.7$ & $8.9 \pm 7.2$ & $8.4 \pm 6.4$ & NS \\
\hline Androstendione $(\mathrm{nmol} / \mathrm{l})$ & $3.7 \pm 1.6$ & $4.1 \pm 1.8$ & $4.3 \pm 1.8$ & NS \\
\hline Estradiol $(\mathrm{pmol} / \mathrm{l})$ & $225.8 \pm 170.7$ & $243.2 \pm 168.5$ & $222.2 \pm 138.9$ & NS \\
\hline $\mathrm{LH}(\mathrm{U} / \mathrm{l})$ & $9.2+5.4$ & $11.1+7.6$ & $11.3+8.4$ & NS \\
\hline $\mathrm{FSH}(\mathrm{U} / \mathrm{I})$ & $4.3 \pm 1.6$ & $4.9 \pm 2.0$ & $4.7 \pm 1.7$ & NS \\
\hline DHEAS $(\mu \mathrm{mol} / \mathrm{l})$ & $0.52 \pm 0.27$ & $0.56 \pm 0.28$ & $0.56 \pm 0.32$ & NS \\
\hline Hirsutsim score & $8.9 \pm 6.4$ & $9.6 \pm 6.5$ & $10.6 \pm 7.4$ & NS \\
\hline Oligomenorrhoea & $50(68 \%)$ & $98(\overline{7} 2 \%)$ & $48(\overline{70} \%)$ & NS \\
\hline Amenorrhoea & $23(32 \%)$ & $38(28 \%)$ & $21(30 \%)$ & NS \\
\hline Acne & $26(36 \%)$ & $54(40 \%)$ & $21(30 \%)$ & NS \\
\hline Alopecia & $28(38 \%)$ & $35(26 \%)$ & $18(26 \%)$ & NS \\
\hline $\mathrm{PCO}$ & $54(74 \%)$ & $103(76 \%)$ & $51(74 \%)$ & NS \\
\hline
\end{tabular}

FAI, free androgen index; LH, luteinizing hormone; FSH, follicle-stimulating hormone; PCO, polycystic ovaries; NS, not significant.

TT, $2.9 \pm 0.9-1.9 \pm 0.7 \mathrm{nmol} / \mathrm{l})$ in PCOS women, independent of GNAS1 genotype. Interestingly, a trend to greater changes in mean FAI levels was found in TT patients compared with $\mathrm{C}$-allele carriers after 6 months on metformin (TT, $\Delta \mathrm{FAI}-4.06 \pm 5.1$; TC, $\Delta \mathrm{FAI}-2.33 \pm 4.8$; $\mathrm{CC}, \Delta \mathrm{FAI}-1.83 \pm 5.2$ ). The improvement of hyperandrogenism remained significant when the subgroup of lean PCOS women was analyzed (CC, testosterone $2.4 \pm 1.0-1.8 \pm 0.9 \mathrm{nmol} / \mathrm{l}, \quad P=0.0389$; TC, testosterone $\quad 3.1 \pm 1.0-2.0 \pm 0.7 \mathrm{nmol} / \mathrm{l}$, $P=0.0037 ; \quad$ TT, testosterone $2.9 \pm 1.5-1.6 \pm 0.5$, $P=0.044)$. Acne improved significantly in all PCOS women (number of affected women: CC, 12 before and 4 after treatment; TC, 10 before and 3 after treatment; TT, 21 before and 8 after treatment). Hirsutism and alopecia did not improve significantly within 6 months of treatment (data not shown). Menstrual cyclicity improved in more than half of the PCOS women studied, independent of the T393C GNAS1 polymorphism (Fig. 2). Furthermore, HOMA-IR (Fig. 3) and indices of insulin sensitivity (data not shown) improved significantly independent of GNAS1 genotype. In addition, mean body weight decreased significantly during treatment in all genotype groups (Fig. 4). Multivariate analysis confirmed that weight reduction was independent of genotype and age but depended on the body weight at baseline. When the $5 \%$ weight loss after 6 months was analyzed in the entire cohort, significant differences were found between $C C$ and TT genotypes $(P<0.05)$ by $\chi^{2}$ testing, but failed to reach the level of statistical significance when an analysis

Table 3 Metabolic characteristics of PCOS women by GNAS1 genotype. Values are presented as mean \pm s.D.

\begin{tabular}{|c|c|c|c|c|}
\hline & $\operatorname{CC}(n=73)$ & $\mathrm{TC}(n=136)$ & TT $(n=69)$ & $\boldsymbol{P}$ \\
\hline Age (years) & $27.9 \pm 6.5$ & $27.6 \pm 5.9$ & $27.8 \pm 5.8$ & NS \\
\hline Body weight (kg) & $90.1 \pm 27.8$ & $87.4 \pm 26.8$ & $80.5 \pm 19.2$ & $0.026^{*}$ \\
\hline BMI $\left(\mathrm{kg} / \mathrm{m}^{2}\right)$ & $31.6 \pm 9.2$ & $31.0 \pm 8.9$ & $28.7 \pm 6.9$ & $0.044^{\dagger}$ \\
\hline Waist circumference $(\mathrm{cm})$ & $95.3 \pm 20.9$ & $94.3 \pm 20.7$ & $89.0 \pm 14.9$ & $N S^{\ddagger}$ \\
\hline HOMA-IR & $4.5 \pm 4.1$ & $4.0 \pm 3.8$ & $3.1 \pm 2.3$ & 0.023 \\
\hline QUICKI & $0.327 \pm 0.037$ & $0.332 \pm 0.041$ & $0.341 \pm 0.042$ & NS \\
\hline Bennett index & $0.1406 \pm 0.0153$ & $0.1439 \pm 0.0173$ & $0.1492 \pm 0.0183$ & $\mathrm{NS}^{\S}$ \\
\hline Matsuda index & $3.9 \pm 3.1$ & $4.9 \pm 4.1$ & $5.5 \pm 4.9$ & $N S^{\S}$ \\
\hline Fasting insulin (pmol/l) & $107.6 \pm 104.5$ & $99.7 \pm 95.3$ & $75.3 \pm 51.1$ & 0.040 \\
\hline AUC insulin & $306.9 \pm 213.8$ & $317.2 \pm 310.7$ & $273.1 \pm 206.5$ & NS \\
\hline Fasting glucose $(\mathrm{mmol} / \mathrm{l})$ & $4.9 \pm 0.6$ & $4.9 \pm 0.7$ & $4.9 \pm 0.6$ & NS \\
\hline 2 -h glucose $(\mathrm{mmol} / \mathrm{l})$ & $5.9 \pm 1.9$ & $5.9 \pm 2.0$ & $5.4 \pm 1.4$ & NS \\
\hline AUC glucose & $19.1 \pm 4.7$ & $19.0 \pm 4.8$ & $18.1 \pm 3.5$ & NS \\
\hline HOMA-B (\%) & $260.6 \pm 175.9$ & $248.2 \pm 184.9$ & $209.5 \pm 113.5$ & $\mathrm{NS}^{\ddagger}$ \\
\hline Total cholesterol $(\mathrm{mmol} / \mathrm{l})$ & $5.0 \pm 0.8$ & $5.0 \pm 1.1$ & $5.0 \pm 1.0$ & NS \\
\hline LDL cholesterol ( $\mathrm{mmol} / \mathrm{l})$ & $3.2 \pm 1.0$ & $3.1 \pm 1.0$ & $3.2 \pm 1.2$ & NS \\
\hline HDL cholesterol $(\mathrm{mmol} / \mathrm{l})$ & $1.5 \pm 0.5$ & $1.4 \pm 0.5$ & $1.5 \pm 0.5$ & NS \\
\hline Triglycerides $(\mathrm{mmol} / \mathrm{l})$ & $1.3 \pm 0.6$ & $1.3 \pm 0.9$ & $1.4 \pm 0.9$ & NS \\
\hline Leptin $(\mathrm{ng} / \mathrm{ml})$ & $35.5 \pm 25.2$ & $36.6 \pm 24.2$ & $26.1 \pm 17.6$ & $0.026^{\| 1,9}$ \\
\hline Ghrelin (pg/ml) & $77.6 \pm 53.7$ & $74.7 \pm 60.6$ & $93.7 \pm 60.2$ & NS \\
\hline
\end{tabular}

BMI, body mass index; HOMA-IR, homeostatic model assessment of insulin resistance; QUICKI, quantitative insulin-sensitivity check index; AUC, area under the curve; HOMA- $\beta$, HOMA of $\beta$-cell function; LDL, low density lipoprotein; HDL, high density lipoprotein; NS, not significant. Statistical analysis by ANOVA or Kruskal-Wallis test as appropriate. * ${ }^{*}$ ignificant differences by Tukey test CC vs TT, $P=0.042$, ${ }^{\dagger}$ Significant differences by Tukey test CC vs TT $P=0.034$, ${ }^{\ddagger}$ Not significant by measurements of ANOVA but significant differences between CC- and TT-women measured by unpaired $t$-test, $P<0.05$. ${ }^{\S}$ Not significant by Kruskal-Wallis test but significant differences between CC- and TT-women measured by Wilcoxon rank sum test, $P<0.05$. "Significant differences by Tukey test CC vs TC, $P=0.033$, "Significant differences by Tukey test CC vs TT, $P=0.021$. 


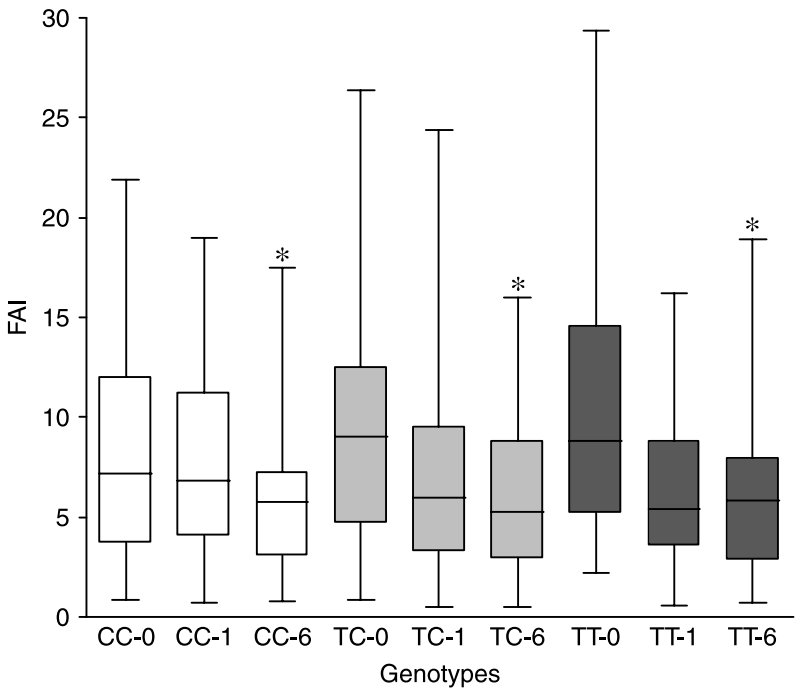

Figure 1 Changes in free androgen index (FAI) during metformin treatment in PCOS patients. The FAI was measured in 105 PCOS women before (0) and after 1 and 6 months of metformin and grouped according to GNAS1 genotype (CC, TC, TT). The decrease was significant in all PCOS subgroups by Wilcoxon sign rank test (CC, $P=0.0271$; TC, $P<0.0001 ; \mathrm{TT}, P=0.0005$ ). Data are shown by box and whiskers graph. The box extends from the 25th to the 75th percentile, with a line at the median indicating the 50 th percentile. The whiskers represent the ranges extending from the lowest to the highest value.

of the three genotype groups was performed. A $5 \%$ weight loss after 6 months was found in 15 out of $28 \mathrm{CC}$ women (53.6\%), 22 out of $51 \mathrm{TC}(43.1 \%)$, and 7 out of 26 TT PCOS patients (26.9\%).

\section{Discussion}

G-protein mutations may cause either loss or gain of function by inactivating or activating signal transduction,

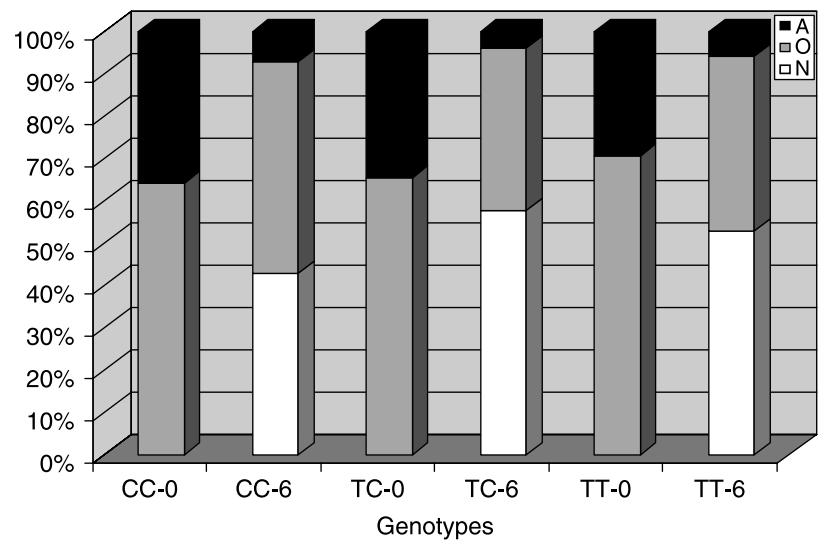

Figure 2 Menstrual cyclicity during metformin treatment in PCOS women. Menstrual cyclicity was recorded in 105 PCOS women before (0) and after 6 months of metformin treatment and grouped according to GNAS1 T393C genotype (CC, TC, TT). The percentage of women with amenorrhea $(A)$ is shown by black, the percentage with those oligomenorrhea $(\mathrm{O})$ by gray and those with normal mentrual cyclicity $(\mathrm{N})$ by white bars.

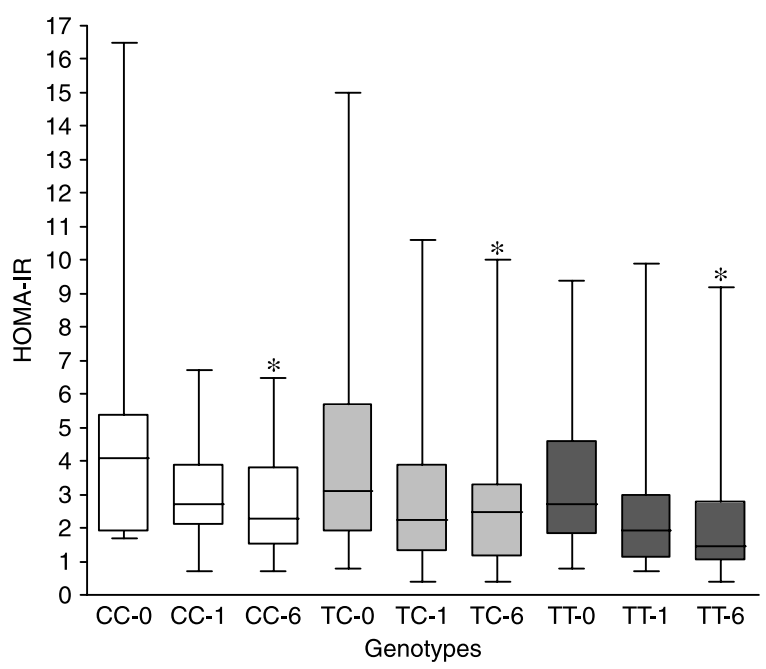

Figure 3 Changes in insulin resistance during metformin treatment in PCOS patients. homeostatic model of insulin resistance (HOMAIR) was measured in 105 PCOS women before (0) and after 1 and 6 months of metformin and grouped according to GNAS1 genotype (CC, TC, TT). The decrease was significant by Wilcoxon sign rank test in all PCOS genotype groups (CC, $P=0.006$; TC, $P<0.0001$; TT, $P=0.0001$ ). Data are shown by box and whiskers graph. The box extends from the 25th to the 75th percentile, with a line at the median indicating the 50th percentile. The whiskers represent the ranges extending from the lowest to the highest value.

thus leading to the clinical phenotype of either hormone deficiency or excess. We were interested in the question whether a variation in the gene encoding for the Gas subunit influences susceptibility to PCOS, a disease characterized by androgen excess.

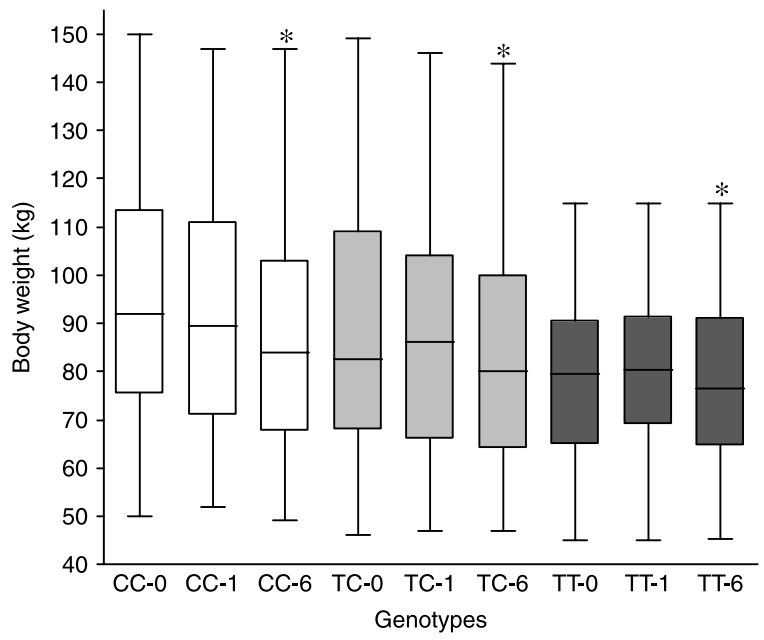

Figure 4 Weight change during metformin treatment in PCOS patients. Body weight was measured in 105 PCOS women before (0) and after 1 and 6 months of metformin treatment and grouped according to GNAS1 genotype (CC, TC, TT). The decrease was found to be significant by paired $t$-test in all groups after 6 months of treatment (CC, $P<0.0001$; TC, $P<0.0001$; TT, $P=0.0475$ ). Data are shown by box and whiskers graph. The box extends from the 25th to the 75th percentile, with a line at the median indicating the 50 th percentile. The whiskers represent the ranges extending from the lowest to the highest value. 
In our German PCOS study cohort, genotype distribution of the T393C polymorphism of the GNAS1 was in accordance with previous studies of other disease entities $(28,36,37)$. In addition, there was no association of the GNAS1 polymorphism with any endocrine clinical and biochemical variable in PCOS women, thus suggesting that the $\mathrm{T} 393 \mathrm{C}$ polymorphism does not contribute to the etiology of PCOS. However, in contrast to healthy control women, the PCOS study group of C-allele carriers showed significantly higher mean body weight, BMI values, indices of insulin resistance, and leptin levels. The gene encoding the Gas subunit (GNAS1) is located at chromosome 20q13.2-13.3. Data showing that this locus is linked to obesity and total energy intake (18) seem to be in accordance with our observation of altered BMI values in PCOS patients. Subgroup analyses of lean, overweight, and obese women showed significant differences in the genotype distribution according to BMI values, possibly in concert with environmental or behavioral factors. Such a gene-environment interaction has been shown for a polymorphism in the gene encoding the $\beta 2$-adrenoceptor, which exerts no effect on BMI in individuals with regular physical activity (38). While the percentage of women who regularly exercised did not differ between the genotype groups, we did not record the frequency and extend of physical activity and, thus, cannot exactly address this question.

The specific role of the T393C polymorphism in obesity is unclear. The few studies on transgenic animals or cell lines focus on the role of $\mathrm{G}$ proteins in adipogenesis. Su et al. (39) demonstrated that increasing Gi $\alpha 2$ activity promotes adipogenic conversion, thus implicating G-proteins as possible regulators of adipogenesis. In humans, no studies on the molecular mechanisms of GNAS1 in obesity are available. The T393C polymorphism is silent, not changing the amino acid of the affected codon in exon 5 of the GNAS1 gene. However, we have previously shown that the GNAS1 polymorphism is associated with altered Gas mRNA expression in different tissues (35). We found that the $\mathrm{T}>\mathrm{C}$ substitution at position 393 changes the mRNA folding structures (37). Therefore, genotype-dependent differences in mRNA decay due to altered secondary structure could finally cause differences in Gas mRNA expression. Nevertheless, a genetic alteration in the regulatory sequences of GNAS1, e.g. in the promotor, which could be in linkage disequilibrium with the T393 $\mathrm{C}$ polymorphism and, which would affect transcription and expression of Gas cannot be excluded.

The entire PCOS cohort and the Metformin-treated subgroup were comparable with those from other Caucasian populations (40) with regard to typical PCOS features, thus a selection bias for the metformin group is unlikely. Our study findings are in agreement with previously published data, showing that metformin improves hyperandrogenism, menstrual cyclicity, and insulin resistance in PCOS women, independent of obesity
(41), especially, when metformin in weight-adapted doses is used.

In clinical practice, interindividual variation in response to metformin is evident. In this context, another aim of the present study was to evaluate whether the T393C polymorphism in GNAS1 may be predictive for changes in endocrine and metabolic parameters by metformin treatment. Recently, Jia et al. suggested that the GNAS1 locus may carry a functional variant that influences response to $\beta$-blockade in essential hypertension (28). In PCOS women, the effect of metformin treatment was shown to be influenced by the IRS-1 Gly972Arg polymorphism (29). In metformin-treated German PCOS patients, no significant association between the GNAS1 T393C polymorphism and treatment outcome with regard to endocrine variables was found. In addition, while there was a trend towards a greater weight reduction in C-allele carriers, multivariate analysis showed that weight loss was predicted by the initial body weight and not by GNAS1 genotype.

In conclusion, the presence of the GNAS1 T393C polymorphism is not associated with PCOS, but seems to influence the degree of obesity and insulin resistance in affected women. Further studies are required to clarify the molecular mechanism that underlie these findings.

\section{References}

1 Legro RS, Bentley-Lewis R, Driscoll D, Wang SC \& Dunaif A. Insulin resistance in the sisters of women with polycystic ovary syndrome: association with hyperandrogenemia rather than menstrual irregularity. Journal of Clinical Endocrinology and Metabolism 200287 2128-2133.

2 Urbanek M, Legro RS, Driscoll DA, Azziz R, Ehrmann DA, Norman RJ, Strauss JF III, Spielman RS \& Dunaif A. Thirty-seven candidate genes for polycystic ovary syndrome: strongest evidence for linkage is with follistatin. PNAS $1999968573-8578$.

3 Xita N, Georgiou I \& Tsatsoulis A. The genetic basis of polycystic ovary syndrome. European Journal of Endocrinology 2002147 717-725.

4 Carmina E. Genetic and environmental aspect of polycystic ovary syndrome. Journal of Endocrinological Investigation 2003 26 1151-1159.

5 Ehrmann DA, Schwarz PE, Hara M, Tang X, Horikawa Y, Imperial J, Bell GI \& Cox NJ. Relationship of calpain-10 genotype to phenotypic features of polycystic ovary syndrome. Journal of Clinical Endocrinology and Metabolism. 200287 1669-1673.

6 Hahn S, Fingerhut A, Khomtsiv U, Khomtsiv L, Tan S, Quadbeck B, Herrmann BL, Knebel B, Muller-Wieland D, Mann K \& Janssen OE. The peroxisome proliferator activated receptor gamma Pro12Ala polymorphism is associated with a lower hirsutism score and increased insulin sensitivity in women with polycystic ovary syndrome. Clinical Endocrinology 200562 573-579.

7 Vrbikova J, Cibula D, Dvorakova K, Stanicka S, Sindelka G, Hill M, Fanta M, Vondra K \& Skrha J. Insulin sensitivity in women with polycystic ovary syndrome. Journal of Clinical Endocrinology and Metabolism $2004892942-2945$.

8 Morin-Papunen LC, Vauhkonen I, Koivunen RM, Ruokonen A \& Tapanainen JS. Insulin sensitivity, insulin secretion, and metabolic and hormonal parameters in healthy women and women with polycystic ovarian syndrome. Human Reproduction $2000151266-1274$. 
9 Goodarzi MO, Erickson S, Port SC, Jennrich RI \& Korenman SG. Relative impact of insulin resistance and obesity on cardiovascular risk factors in polycystic ovary syndrome. Metabolism 200352 713-719.

10 Hahn S, Tan S, Elsenbruch S, Quadbeck B, Herrmann BL, Mann K \& Janssen OE. Clinical and biochemical characterization of women with polycystic ovary syndrome in North Rhine-Westphalia. Hormone and Metabolic Research 200537 438-444.

11 Comuzzie AG \& Allison DB. The search for human obesity genes. Science $19982801374-1377$.

12 Buono P, Pasanisi F, Nardelli C, Ieno L, Capone S, Liguori R, Finelli C, Oriani G, Contaldo F \& Sacchetti L. Six novel mutations in the proopiomelanocortin and melanocortin receptor 4 genes in severely obese adults living in southern Italy. Clinical Chemistry 200551 1358-1364.

13 Clement K, Vaisse C, Lahlou N, Cabrol S, Pelloux V, Cassuto D, Gourmelen M, Dina C, Chambaz J, Lacorte JM, Basdevant A, Bougneres P, Lebouc Y, Froguel P \& Guy-Grand B. A mutation in the human leptin receptor gene causes obesity and pituitary dysfunction. Nature $1998 \mathbf{3 9 2} 398-401$.

14 Conneely KN, Silander K, Scott LJ, Mohlke KL, Lazaridis KN, Valle TT, Tuomilehto J, Bergman RN, Watanabe RM, Buchanan TA, Collins FS \& Boehnke M. Variation in the resistin gene is associated with obesity and insulin-related phenotypes in Finnish subjects. Diabetologia 200447 1782-1788.

15 Menzaghi C, Ercolino T, Di Paola R, Berg AH, Warram JH, Scherer PE, Trischitta V \& Doria A. A haplotype at the adiponectin locus is associated with obesity and other features of the insulin resistance syndrome. Diabetes 200251 2306-2312.

16 Siffert W, Forster P, Jockel KH, Mvere DA, Brinkmann B, Naber C, Crookes R, Du PHA, Epplen JT, Fridey J, Freedman BI, Muller N, Stolke D, Sharma AM, Al Moutaery K, Grosse-Wilde H, Buerbaum B, Ehrlich T, Ahmad HR, Horsthemke B, Du Toit ED, Tiilikainen A, Ge J, Wang Y, Yang D, Hüsing J \& Rosskopf D. Worldwide ethnic distribution of the $\mathrm{G}$ protein beta 3 subunit $825 \mathrm{~T}$ allele and its association with obesity in Caucasian, Chinese, and Black African individuals. Journal of the American Society of Nephrology 199910 1921-1930.

17 Escobar-Morreale HF, Calvo RM, Villuendas G, Sancho J \& San Millan JL. Association of polymorphisms in the interleukin 6 receptor complex with obesity and hyperandrogenism. Obesity Research 200311 987-996.

18 Lee JH, Reed DR, Li WD, Xu W, Joo EJ, Kilker RL, Nanthakumar E, North M, Sakul H, Bell C \& Price RA. Genome scan for human obesity and linkage to markers in 20q13. American Journal of Human Genetics 199964 196-209.

19 Cummings DE, Brandon EP, Planas JV, Motamed K, Idzerda RL \& McKnight GS. Genetically lean mice result from targeted disruption of the RII beta subunit of protein kinase A. Nature $1996382622-626$.

20 Freudzon L, Norris RP, Hand AR, Tanaka S, Saeki Y, Jones TL, Rasenick MM, Berlot CH, Mehlmann LM \& Jaffe LA. Regulation of meiotic prophase arrest in mouse oocytes by GPR3, a constitutive activator of the Gs G protein. Journal of Cell Biology 2005171 255-265.

21 Mehlmann LM, Jones TL \& Jaffe LA. Meiotic arrest in the mouse follicle maintained by a Gs protein in the oocyte. Science $2002 \mathbf{2 9 7}$ 1343-1345.

22 Mehlmann LM, Saeki Y, Tanaka S, Brennan TJ, Evsikov AV, Pendola FL, Knowles BB, Eppig JJ \& Jaffe LA. The Gs-linked receptor GPR3 maintains meiotic arrest in mammalian oocytes. Science 2004306 1947-1950.

23 Fragoso MC, Latronico AC, Carvalho FM, Zerbini MC, Marcondes JA, Araujo LM, Lando VS, Frazzatto ET, Mendonca BB \& Villares SM. Activating mutation of the stimulatory G protein (gsp) as a putative cause of ovarian and testicular human stromal Leydig cell tumors. Journal of Clinical Endocrinology and Metabolism $1998832074-2078$.

24 Fragoso MC, Domenice S, Latronico AC, Martin RM, Pereira MA, Zerbini MC, Lucon AM \& Mendonca BB. Cushing's syndrome secondary to adrenocorticotropin-independent macronodular adrenocortical hyperplasia due to activating mutations of GNAS1 gene. Journal of Clinical Endocrinology and Metabolism 200388 2147-2151.

25 Roman R, Johnson MC, Codner E, Boric MA, aVila A \& Cassorla F. Activating GNAS1 gene mutations in patients with premature thelarche. Journal of Pediatrics $2004145218-222$.

26 Chen M, Haluzik M, Wolf NJ, Lorenzo J, Dietz KR, Reitman ML \& Weinstein LS. Increased insulin sensitivity in paternal Gnas knockout mice is associated with increased lipid clearance. Endocrinology 2004145 4094-4102.

27 Weinstein LS, Yu S, Warner DR \& Liu J. Endocrine manifestations of stimulatory $\mathrm{G}$ protein alpha-subunit mutations and the role of genomic imprinting. Endocrine Reviews 200122 675-705.

28 Jia H, Hingorani AD, Sharma P, Hopper R, Dickerson C, Trutwein D, Lloyd DD \& Brown MJ. Association of the G(s)alpha gene with essential hypertension and response to beta-blockade. Hypertension $1999348-14$.

29 Ertunc D, Tok EC, Aktas A, Erdal EM \& Dilek S. The importance of IRS-1 Gly972Arg polymorphism in evaluating the response to metformin treatment in polycystic ovary syndrome. Human Reproduction 200520 1207-1212.

30 ESHRE/ASRM. Revised 2003 consensus on diagnostic criteria and long-term health risks related to polycystic ovary syndrome. Fertility and Sterility 200481 19-25.

31 Matthews DR, Hosker JP, Rudenski AS, Naylor BA, Treacher DF \& Turner RC. Homeostasis model assessment: insulin resistance and beta-cell function from fasting plasma glucose and insulin concentrations in man. Diabetologia $1985 \mathbf{2 8} 412-419$.

32 Katz A, Nambi SS, Mather K, Baron AD, Follmann DA, Sullivan G \& Quon MJ. Quantitative insulin sensitivity check index: a simple, accurate method for assessing insulin sensitivity in humans. Journal of Clinical Endocrinology and Metabolism 200085 2402-2410.

33 Radikova Z. Assessment of insulin sensitivity/resistance in epidemiological studies. Endocrine Regulations 200337 189-194.

34 Matsuda M \& DeFronzo RA. Insulin sensitivity indices obtained from oral glucose tolerance testing: comparison with the euglycemic insulin clamp. Diabetes Care 199922 1462-1470.

35 Frey UH, Eisenhardt A, Lummen G, Rubben H, Jockel KH, Schmid KW \& Siffert W. The T393C polymorphism of the G alpha s gene (GNAS1) is a novel prognostic marker in bladder cancer. Cancer Epidemiology, Biomarkers and Prevention $200514871-877$.

36 Abe M, Nakura J, Yamamoto M, Jin JJ, Wu Z, Tabara Y, Yamamoto Y, Igase M, Kohara K \& Miki T. Association of GNAS1 gene variant with hypertension depending on smoking status. Hypertension $2002 \mathbf{4 0} 261-265$.

37 Frey UH, Alakus H, Wohlschlaeger J, Schmitz KJ, Winde G, van Calker HG, Jockel KH, Siffert W \& Schmid KW. GNAS1 T393C polymorphism and survival in patients with sporadic colorectal cancer. Clinical Cancer Research $2005115071-5077$.

38 Meirhaeghe A, Helbecque N, Cottel D \& Amouyel P. Beta2adrenoceptor gene polymorphism, body weight, and physical activity. Lancet $19993 \mathbf{3 5 3} 896$.

$39 \mathrm{Su}$ HL, Malbon CC \& Wang HY. Increased expression of Gi alpha 2 in mouse embryo stem cells promotes terminal differentiation to adipocytes. American Journal of Physiology 1993265 C1729-C1735.

40 Wijeyaratne CN, Balen AH, Barth JH \& Belchetz PE. Clinical manifestations and insulin resistance (IR) in polycystic ovary syndrome (PCOS) among South Asians and Caucasians: is there a difference? Clinical Endocrinology 200257 343-350.

41 Goldenberg N, Glueck CJ, Loftspring M, Sherman A \& Wang P. Metformin-diet benefits in women with polycystic ovary syndrome in the bottom and top quintiles for insulin resistance. Metabolism $200554113-121$.

Received 7 May 2006

Accepted 29 August 2006 